\title{
Whey hydrolysate formula reduced crying time in infantile colic
}

\author{
Lucassen PL, Assendelft WJ, Gubbels JW, et al. Infantile colic: crying time reduction with a whey hydrolysate: a double-blind, \\ randomized, placebo-controlled trial. Pediatrics 2000 Dec;106:1349-54.
}

\section{QUESTION: Is whey hydrolysate formula effective in the treatment of infantile colic in formula fed infants in a primary care setting?}

Design

Randomised (allocation concealed), blinded (research nurses and parents), placebo controlled trial with follow up immediately after 1 week of intervention.

\section{Setting}

Community based, well baby clinics in 6 regions of the Netherlands.

\section{Patients}

43 healthy, thriving, formula fed $(\geqslant 1$ formula feeding per d) infants $<6$ months old who cried for $>3$ hours/ day on $\geqslant 3$ days/week (average $>4.5 \mathrm{~h} / \mathrm{d}$ ). Exclusion criteria included participation in a previous trial of hypoallergenic feeding, anaphylactic reactions to cows' milk, and non-Dutch speaking parents. 5 infants (12\%) did not complete the trial but were included in the analysis. For the 38 who did complete the trial, $53 \%$ were boys and the mean weight was $4.7 \mathrm{~kg}$.

\section{Intervention}

After a 1 week qualification period, 23 infants were allocated to whey hydrolysate formula (Nutrilon Pepti) and 20 to standard cows' milk formula (Nutrilon Premium) for a 1 week intervention period. Every infant was provided with 2 cans of formula powder, each can con- taining $400 \mathrm{~g}$. To make the taste, smell, and appearance of the 2 formulas similar, a quarter of the powder in each can of standard formula was substituted with hydrolysate powder.

\section{Main outcome measures}

The primary outcome was difference in duration of crying $(\mathrm{min} / \mathrm{d})$ between the qualification week and intervention week. The secondary outcome was proportion of infants no longer fulfilling the inclusion criteria (ie, having infantile colic).

\section{Main results}

Analysis was by intention to treat. The crying of infants allocated to whey hydrolysate formula decreased by 63 minutes/day (when adjusted for sex and amount of crying before intervention) more than the crying of infants allocated to standard formula $(\mathrm{p}=0.05)$. Of those completing the trial (38 infants, $88 \%$ ), the proportion of infants no longer fulfilling the inclusion criteria did not differ between the 2 groups (whey hydrolysate $40 \% v$ standard formula $28 \%, p=0.65$ ).

\section{Conclusion}

In formula fed infants with infantile colic, crying time was reduced with whey hydrolysate formula.
Sources of funding: Praeventie Fonds; formulas provided by Nutricia.

For correspondence: Dr P L Lucassen, Akkerroosstraat 18, $5761 \mathrm{EX}$, Bakel, the Netherlands. Fax +31 492343975.

\section{COMMENTARY}

Infantile colic is common during the first months of life and, despite extensive study, its cause remains unclear. This is reflected in the numerous approaches to treatment that have been recommended and studied. Although its symptoms usually resolve by $4-5$ months of age, colic causes appreciable distress to parents and creates needs for teaching and counselling by nurses. It is therefore an important issue for antepartum and postpartum nurses working in hospital and community settings.

The 2 studies presented here used the same diagnostic definition of infantile colic. ${ }^{1}$ The infants in both studies and in both groups, however, had very high levels of crying $(>4.5 \mathrm{~h} / \mathrm{d}$ compared with the diagnostic cut off of $>3 \mathrm{~h} / \mathrm{d}$, for $>3 \mathrm{~d} / \mathrm{wk}$ ). This initially high crying time made it more difficult to achieve large enough reductions to meet one of the outcome criteria: a reduced proportion of infants meeting the diagnostic definition of colic. The high crying time among study participants also may reflect the presence of other confounding factors.

In the study of chiropractic treatment by Olafsdottir et al, there was reduced infant crying and parents reported an improvement in both the intervention and placebo groups, with no statistically significant differences between them. The similar improvement may be the result of a placebo effect (ie, the counselling and support provided to parents in both groups) and/or the natural course of infantile colic. The lack of effect from spinal manipulation differs from other trial results, possibly because this study had the critical methodological strength of blinding the parents to the intervention, minimising the potential for bias arising from parental expectations. These findings are important in locales where chiropractic treatment is an accepted treatment for colic and nurses are asked for advice about this treatment.

The study of whey hydrolysate by Lucassen $e t$ al presents results that are consistent with findings from a systematic review of treatments for colic, which concluded that replacement of cows' milk protein with a hydrolysate is effective. ${ }^{2}$ It is not clear, however, how much formula the infants in this study consumed because the inclusion criterion was at least 1 formula feeding a day, and the amount of breast feeding in the sample group, before and during the study, was not reported. It is important to know the extent of breast feeding in the 2 groups as this may have affected the differences in outcomes. It is also not stated whether only formula feedings, or breast milk feedings as well, were replaced with whey hydrolysate. This limits the generalisability of results, and does not provide any evidence to support replacement of breast milk with whey hydrolysate formula.

continued on next page 